\title{
This Is How To Be a Rule Breaker
}

\author{
Robert Wirth, Anna Foerster, Oliver Herbort, Wilfried Kunde, and Roland Pfister
}

Department of Psychology, Julius-Maximilians-University of Würzburg, Germany

ABSTRACT

Violating rules comes with cognitive conflict for the rule-breaker. Here, we probed for means to reduce the behavioral effects of this conflict by studying the combined impact of recency and frequency of rule violations. We found that violating a rule facilitated the initiation of a subsequent rule violation, while notable costs relative to rule-based responding remained in measures of response execution. Such costs during response execution vanished, however, when frequency and recency of rule violation worked in concert. That is, it is possible to overcome the costs of rule violation when (a) having violated this particular rule frequently and (b) having violated this particular rule very recently. Moreover, we demonstrated that recent rule violations reduce the costs of cognitive conflict in an unrelated interference task (Simon task). Based on these findings, we present a revised model of the cognitive processes underlying deliberate rule violations.

\section{INTRODUCTION}

\section{Rules}

Life could be so easy. When we are late, we could just speed and run red lights with no regard for the other motorists, cyclists, and pedestrians. "Advance to Go, collect 200." When our bank account is empty, we could commit tax fraud to improve our finances. "Income tax refund, collect 20." We could cheat, lie, litter, jaywalk, steal, hack, swindle, infringe, and deceive to solve our problems. "You have won a crossword competition, collect 100." But by and large, we do not. Instead, we wait at a red traffic light, do our taxes properly, carry our trash to the next trashcan, return lost property, and download music legally. Granted, most of us avoid the initially described way of life because it usually comes with consequences that may be enforced by authorities (Wirth, Foerster, Rendel, Kunde, \& Pfister, 2017). "Go directly to jail, do not pass Go, do not collect 200." However, even in the absence of punishment, people tend to adhere to rules (Pfister, Wirth, Schwarz, Steinhauser, \& Kunde, 2016; Wirth, Pfister, Foerster, Huestegge, \& Kunde, 2016). These rules do not even have to be explicit to invoke compliance (Asch, 1956), and at times the confrontation with an imperative rule can even annul personal values (Milgram, 1963). Adherence to rules also comes with positive side-effects, as rule-compliant individuals are viewed as trustworthy and good social partners (Everett, Pizarro, \& Crockett, 2016). Equally, honest individuals are judged as more attractive and healthier than dishonest individuals (Paunonen, 2006). Such positive side-effects even seem to be sufficiently adaptive to have rendered rulebased behavior an evolutionary default (Hoffman, 1981). People like people who stick to the rules. And an evolutionary advantage for those who adhere to rules might be a driving force that allowed humans to create and maintain complex social structures in the first place.

\section{Rule Violations}

Based on this reasoning, committing a rule violation should not come naturally. But isolating the burdens of nonconformity experimentally can be challenging because, when comparing rule-based behavior to rule violations, in the latter case there are a lot of additional factors

Corresponding author: Robert Wirth, Department of Psychology, JuliusMaximilians-University of Würzburg, Röntgenring 11, 97070 Würzburg Germany. Phone: +49 - (0)931 - 3181855.

Email: robert.wirth@uni-wuerzburg.de 
that might influence the results. Therefore, we conceptualized rule violations as responses that are counterindicated by an instructed but otherwise arbitrary mapping rule. Violating these rules did not have any consequences for the participants, and they did so in a nonsocial setting. This deliberate design choice allowed us to isolate the cognitive architecture that processes (non-)conformity removed from any social influences, prior experience with nonconformity, morals, and expectations of punishment, and left us with a highly controlled experimental setup: Neither following nor breaking the particular rule came with a prior learning history or an avoidance due to punishment. Further, participants were instructed whether to follow or break a rule. Again, this was done to control for the ratio of both response options, and a systematic comparison of participants who could choose freely whether to follow or break a rule to participants who were instructed what to do showed that the signature of nonconformity was uninfluenced by the type of choice (free vs. forced; Pfister, Wirth, Schwarz, Steinhauser, \& Kunde, 2016). To gauge the cognitive mechanism that processes nonconformity (rather than measuring real-life costs of violating real-life rules), in the following studies we opted for the highly controlled approach.

Indeed, empirical evidence shows that, even when removed from all social influences, and even without any negative consequences, following an arbitrary rule is much easier than breaking it. Further, a response that is labeled as a rule violation is harder to carry out than an identical response that is labeled with a more neutral term, such as rule inversion (although both require the same cognitive and motor operations, Wirth, Pfister, Foerster, et al., 2016). Specifically, participants in this line of experiments either followed or broke an instructed rule by moving the finger to one of two predefined areas on a touchscreen. Violating a rule consistently decreased performance: Rule violations were initiated and executed more slowly than rule-based responses, and the movement trajectories of violations were markedly attracted to the alternative, rule-based target (note that, throughout this paper, rule-based refers to the instructed mapping rule, violation refers to the breaking of the instructed rule, even when prompted externally). We reasoned that these effects emerged because breaking a rule requires overcoming an initially rule-based tendency and thus executive control.

\section{Reducing the Effects of Nonconformity}

Overall, the empirical evidence suggests that cognitive costs are an inevitable burden of rule violations. However, there are individuals who might be more efficient than others at violating rules. Take, for example, criminals convicted for theft, fraud, swindle, or forgery. When these individuals are asked to break rules in an experimental setting, they show significantly reduced response costs for violations when compared to a control group with no criminal history (Jusyte et al., 2017). They seem to suffer less from the burdens of nonconformity, which ultimately enables them to break rules more easily (law of less work, Kool, McGuire, Rosen, \& Botvinick, 2010). Equally, lying is considered the socially disregarded alternative to being honest, and for most people, telling lies is associated with cognitive effort (Duran, Dale, \& McNamara, 2010; Foerster, Wirth, Kunde, \& Pfister, 2017; Spence et al., 2001). Still, the majority of lies are told only by a few prolific liars, while most people are honest most of the time (Serota \& Levine, 2014). The enhanced cognitive effort that comes with lying (which might be reduced for prolific liars) could drive our tendency to be customarily honest. Further, some so-called countercultures (e.g., punks) even advertise a sympathy for deviancy and nonconformity, combined with a healthy disrespect for the dominant value system, as their defining feature (Fox, 1987; Yinger, 1982).

What is still unclear is whether individuals who are less subject to the response costs of rule violations are "born this way," with a cognitive system that is hard-wired to be afflicted less by the struggles of overcoming rule-based behavior, or whether they manage to circumvent these burdens by any means. If the latter were true, the following questions are in order: Is there a way to enable anyone to violate rules efficiently without being thwarted by their distinct behavioral signature? What circumstances allow us to become capable and skillful rule breakers?

Aside from curiosity, why would attempts to facilitate rule violation be desirable? Next to the negative examples discussed so far, nonconformity can have an immediate positive spin: Prosocial behavior also falls within the realm of nonconformity, where people do something that is unusual, extraordinary, creative, different from what the others do, where they speak up instead of remaining silent, where they help instead of just standing by (Csíkszentmihályi, 1996; Darley \& Latané, 1968; Dovidio, Piliavin, Schroeder, \& Penner, 2006). In these cases, we also have to overcome our default tendency to adhere to the group norms to give way for the prosocial behavior. Innovation, per definition, includes the deviation from common ways of solving problems as well. So, again: If we broke rules more efficiently, we might more easily behave in a prosocial and innovative manner. In the following experiments, we approach this subject by testing whether response costs for rule violations can be reduced by controlled, situational variations.

\section{EXPERIMENT 1}

\section{Introduction}

Cognitive psychology offers a range of tools to reduce response costs in tasks that recruit executive control, with the most prominent ones being (a) frequent and (b) recent exposure to the cost-invoking conditions in question. Response costs and the factors that modulate them have mainly been studied in conflict paradigms, in which participants have to respond to task-relevant information while avoiding distraction by task-irrelevant information ${ }^{1}$. Performance is typically good (i.e., fast and correct) if task-relevant and task-irrelevant information call for the same response (nonconflicting trials) whereas performance suffers if they call for different responses (conflicting trials). In frequency manipulations, the proportion of conflicting trials is raised, and as a consequence, response costs for the conflicting trials decrease (Logan \& Zbrodoff, 1979). In recency manipulations, conflicting and noncon- 
flicting trials are analyzed as a function of the immediately preceding trial, resulting in reduced response costs in conflicting trials after having just experienced a conflicting trial (Gratton, Coles, \& Donchin, 1992). Both these factors seem to reduce conflict effects independently of each other (Torres-Quesada, Funes, \& Lupiáñez, 2013).

However, there is reason to doubt whether these manipulations also exert their influence on rule violation tasks. The typical adaptation effects to recent conflict were found only for the duration of action planning and response initiation. Response costs for repeatedly initiating a violation were smaller as compared to single violation instances, whereas response execution remained unaffected (Wirth, Pfister, Foerster, et al., 2016): Repeated violation responses turned out to be just as slow and spatially contorted as a single violation, and this pattern is markedly unusual even for response trajectories (cf. Scherbaum, Dshemuchadse, Fischer, \& Goschke, 2010).

Consequently, planning a violation and actually carrying it out might rely on separate cognitive processes. Rule violations are thought to represent a separate task set (Allport, Styles, \& Hsieh, 1994; Monsell, 2003; Rogers \& Monsell, 1995) which, crucially, is not implemented on its own, but is derived by negating the instructed rule (Wirth, Pfister, Foerster, et al., 2016). Switching between task sets usually entails the inhibition of the previously used task set (Koch, Gade, Schuch, \& Philipp, 2010). However, this mechanism would be detrimental here, as the task set for violations heavily relies on the task set for rule-based responses and is not necessarily meaningful on its own (Hasson, Simmons, \& Todorov, 2005). During violations, both task sets are likely held activated, which creates conflict between the two diverging representations (Hsieh, Chang, \& Meiran, 2012; Kuhns, Lien, \& Ruthruff, 2007; Meiran, Hsieh, \& Dimov, 2010). So afterwards, one of the task sets is likely to be inhibited again to resolve such ongoing conflict. As the violation task set cannot be held activated on its own (because it is represented as a transformation of the rule task set), it is automatically inhibited after use. Consequently, a violation arguably includes an immediate, endogenous switch back to the rule-based task set (see also the General Discussion section for a more elaborate model; for literature on endogenous, voluntary task-switching, see Arrington \& Logan, 2004; Kessler, Shencar, \& Meiran, 2009).

Altogether, we describe the production of a rule violation as a twostep process: To violate a rule, the rule is first activated and then modulated (e.g., negated, inverted, reformulated). Based on this new derived task set, a violation response can be selected. Assuming that the original rule is imperatively activated during violations explains why violation responses remain attracted towards the rule-based response (Pfister, Wirth, Schwarz, Steinhauser, \& Kunde, 2016; Wirth, Pfister, Foerster, et al., 2016). While the selection of the violation task set, prior to response initiation, follows common task-switching logic, the implementation of this task set seems to be less efficient: The violation task set has to be reimplemented every single time a violation response is required, resulting in a repeated effort for repeated violations.

If we assume that rule violations are selected via a two-step activation process that generates a derived task set which is inhibited after use, recency adaptations should emerge for response planning (which reflects task set selection: the violation task set is present, but inhibited) but not for response execution (which reflects task set implementation: the task set is inhibited and has to be reimplemented every time). This assumption is backed up by previous findings (Wirth, Pfister, Foerster, et al., 2016), and we will further replicate these results in the present experiments.

In contrast to the recency manipulation, there is currently no empirical evidence for the impact of rule violation frequency on violation performance. Experiment 1 aimed at filling this gap. For response initiation, previous findings and the corresponding two-step model suggest that the effects of a frequency manipulation should be similar to effects of frequency manipulations in other conflict tasks (Logan \& Zbrodoff, 1979). For response execution, the two-step activation model would motivate opposing scenarios. For one, the assumed inhibition of the violation task set after each response would suggest the frequency manipulation to have no effect on response execution. But one might also assume that frequent use of the violation task set reduces self-inhibition after use, to prepare for its frequent future use, which could possibly reverse the violation effect. Speculatively, if a violation task set is used frequently, even response execution could be affected by recency manipulations (in contrast to previous findings). To replicate the impact of violation recency and test for the impact of violation frequency, both manipulations were implemented together in an integrated design to assess their individual contributions, as well as a possible interplay between the two manipulations. We therefore adapted previous methods that targeted the sole impact of violation recency (Wirth, Pfister, Foerster, et al., 2016), and we further added a manipulation of high versus low violation frequency between blocks.

\section{Method}

\section{PARTICIPANTS}

Twenty-four participants were recruited $\left(M_{\text {age }}=26.3\right.$ years, $S D=$ 7.8 , seven male, four left-handed) and received either course credit or $8 €$ monetary compensation. All participants gave informed consent, were naïve to the purpose of the experiment, and were debriefed after the session.

\section{APPARATUS AND STIMULI}

The experiment was modeled after Wirth, Pfister, Foerster, et al., (2016). It was run on an iPad in portrait mode with a viewing distance of about $50 \mathrm{~cm}$. Participants used the index finger of their dominant hand for input on the touchscreen, which sampled the finger movements at $100 \mathrm{~Hz}$. We used two chess symbols (king, and pawn, 踇) as target stimuli to prompt movements to the left or to the right target area (two circles $2 \mathrm{~cm}$ in diameter in the upper left and right corners of the display). The target areas were separated by $11 \mathrm{~cm}$ (center-tocenter). In between trials, the two chess symbols were displayed to the left and right of the screen center to remind participants of the mapping rule. A written instruction between the two chess figures instructed the rule-compliance for the following trial. The starting position for the movement (a circle $1 \mathrm{~cm}$ in diameter) was located at the bottom center 


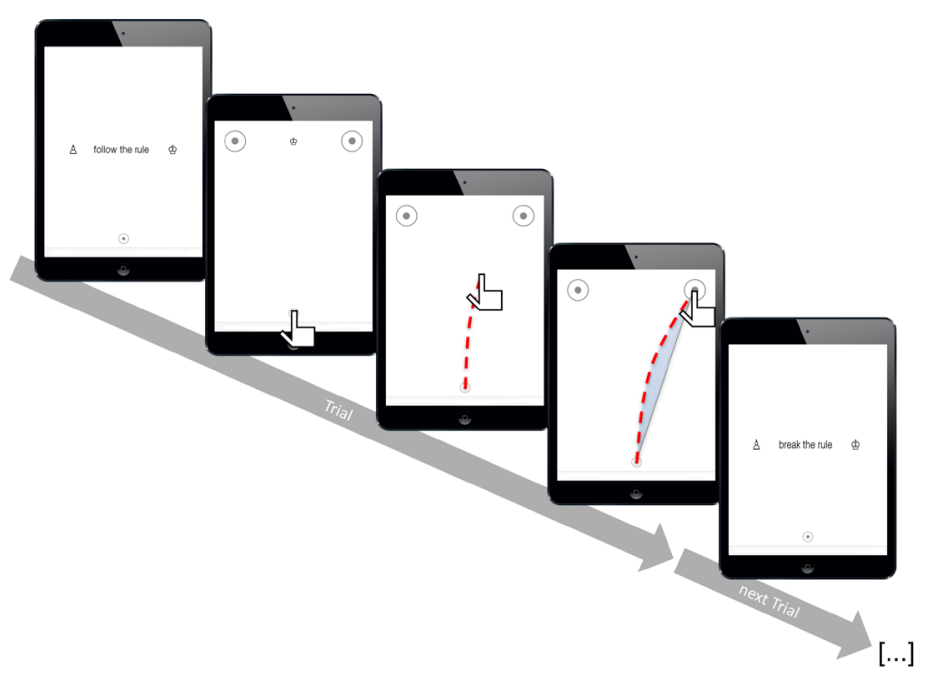

FIGURE 1.

Procedure of the experiments. Before each trial, participants were reminded of the mapping rule, together with the instruction to either follow or break the rule in the upcoming trial. As soon as participants put their finger on the starting area, the mapping rule disappeared and the two target areas and the target symbol appeared, prompting movements to the left or the right. The target symbol disappeared when the finger left the starting area. A trial was completed when the finger was lifted from the screen inside one of the two target areas, and the next trial started immediately with the corresponding instructions to follow or break the rule.

of the screen, $17 \mathrm{~cm}$ from the middle of the two target positions at an angle of $31^{\circ}$ to each side. Stimuli were presented against a white background (see Figure 1).

\section{PROCEDURE}

Before each trial, the stimulus-response (S-R) mapping and a written instruction to either follow (German: "Regel befolgen") or break (German: "Regel brechen") the rule in the next trial was displayed. In rule-violation trials, the displayed mapping rule had to be violated; the response that a target originally required was now contraindicated. Participants started a trial by touching the starting area with the index finger of the dominant hand. Immediately, one of the two target symbols appeared between the two target areas to indicate whether a movement to the left or a movement to the right had to be executed. Simultaneously, the reminder of the S-R mapping rule and the written instruction disappeared. Half of the participants were instructed to make a smooth finger movement to the left target area in response to a pawn symbol and to the right target area in response to a king symbol (cf. Figure 1). The other half of the participants was instructed with the opposite S-R mapping for counterbalancing. The target symbol disappeared as soon as the finger left the starting area. A trial ended when the finger was lifted from the touchscreen. Error feedback was displayed only if participants failed to hit one of the designated target areas. Between blocks, the proportion of violation trials was manipulated: In blocks with a low proportion of rule violations (low-PV), the displayed mapping rule had to be violated in one out of four trials. In blocks with a high proportion of rule violations (high-PV), the mapping rule had to be violated in three out of four trials. The proportion of violations within a block changed after half of the experiment, the order of presentation (first half: low-PV, second half: high-PV vs. first half: high-PV, second half: low-PV) was manipulated between participants. Instructions stressed that responses had to be delivered quickly and accurately; still the experiment was self-paced, so participants chose on their own when to start a trial and how long they took breaks in between blocks. Participants completed 20 blocks of 64 trials each.

\section{Results}

\section{PREPROCESSING}

We analyzed three variables of each movement: The time it took participants to leave the starting area after touching it (initiation time; IT), the duration of the movement after leaving the starting area (movement time; MT), and the area between the actual movement trajectory and a straight line from start- to endpoint (area under the curve; AUC; shaded area in Figure 1). The area under the curve was computed from the time-normalized coordinate data of each trial by using custom MATLAB scripts (The Mathworks, Inc.). Movements to the left were mirrored at the vertical midline for all analyses. The area under the curve was computed as the signed area relative to a straight line from start- to endpoint of the movement. Positive values indicate attraction toward the opposite side (indicating a persisting influence of the original mapping rule in case of rule violations), negative values indicate attraction toward the nearest edge of the display.

To reduce complexity, we focus on MT and AUC in the results sections, as these variables had produced the distinct additive pattern of results that set rule violations apart from other types of cognitive conflict (Wirth, Pfister, Foerster, et al., 2016). The analysis of the initiation times (ITs) can be accessed online (www.osf.io/a2apv). 


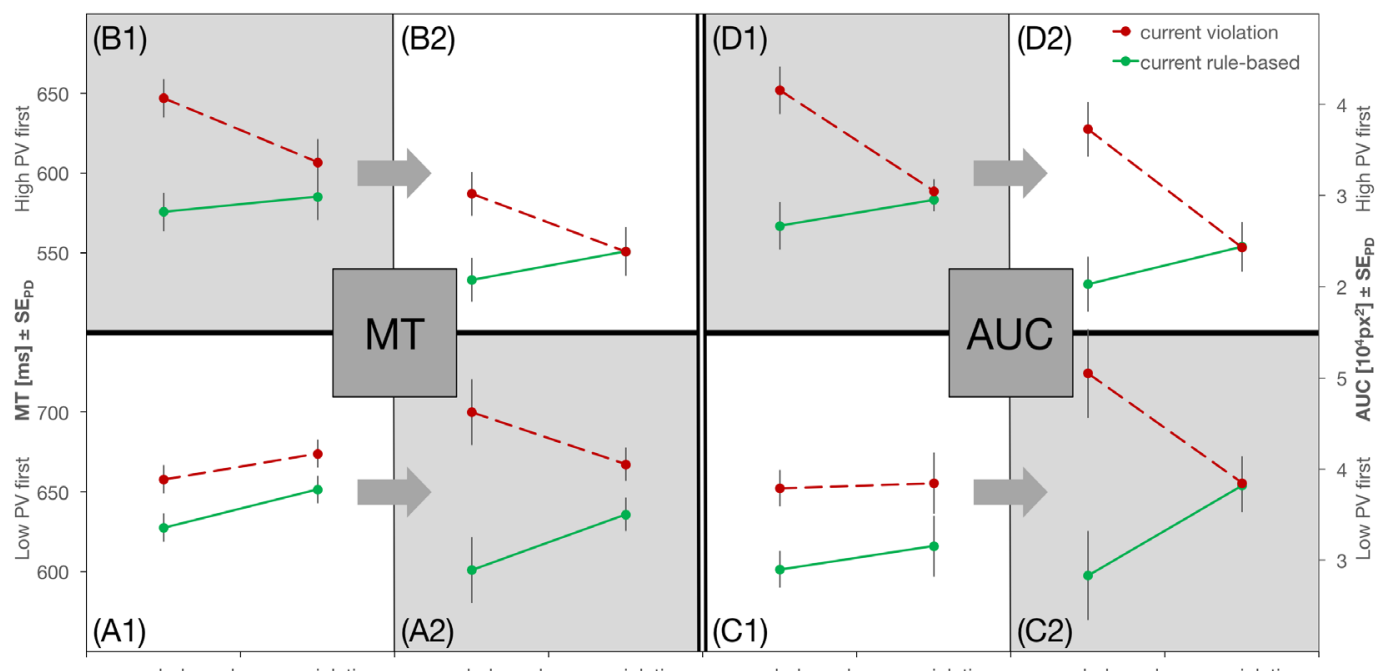

prec. rule-based prec. violation prec. rule-based prec.violation prec. rule-based prec. violation prec. rule-based prec. violation

FIGURE 2.

Results for Experiment 1. Movement times (MT; left) and areas under the curve (AUC; right) are plotted as a function of preceding response type (abscissa), current response type (continuous green line for rule-based responses; dashed red line for violation responses), and the current proportion of violations ( $\mathrm{PV}$; white background for low-PV, gray background for high-PV). Further, the figure is split by proportion order:The lower panels ( $A$ and $C$ ) represent the low-PV-first condition, the upper panels $(B$ and $D)$ represent the high-PV-first condition. Note that scaling of the $y$-axes differs between proportion orders. Panels with the number 1 represent the first half of the experiment per proportion order, panels with the number 2 represent the second half. Error bars represent SEs of paired differences, calculated separately for each instance of preceding response type (Pfister \& Janczyk, 2013).

\section{DATA SELECTION AND ANALYSES}

For all analyses, the first block of each PV condition was considered practice and removed. We then omitted trials in which participants failed to act according to the instruction or failed to hit any of the two target areas at all (6.2\%) and trials following an error (4.8\%). Trials were discarded as outliers if any of the measures (IT, MT, AUC) deviated more than 2.5 SDs from the respective cell mean (5.9\%).

Because initial analyses suggested profound higher-order interactions of proportion order and the remaining variables, we opted to break down this complex pattern by conducting separate $2 \times 2 \times$ 2 analyses of variance (ANOVAs) with current response type (rulebased vs. violation), preceding response type, and proportion violation (low-PV vs. high-PV) as within-subject factors for each condition of proportion order (low-PV-first vs. high-PV-first; see Figure 2). More complex omnibus analyses that include the factor proportion order in the ANOVA model, as well as results regarding ITs, can be found online on the Open Science Framework (see www.osf.io/a2apv).

\section{MOVEMENT TIMES, LOW-PV-FIRST}

A significant effect of current response type, $F(1,11)=53.93, p<$ $.001, \eta_{\mathrm{p}}{ }^{2}=.83$, was driven by slower responses for violations $(665 \mathrm{~ms})$ than for rule-based behavior (622 ms). The interaction between current response type and proportion violation was also significant, $F(1$, 11) $=13.47, p=.004, \eta_{\mathrm{p}}{ }^{2}=.55$, with a stronger effect of violations in high-PV blocks $(\Delta=64 \mathrm{~ms})$ compared to low-PV blocks $(\Delta=21 \mathrm{~ms}$, see Footnote ${ }^{2}$ ). The interaction between preceding response type and current response type was significant, $F(1,11)=5.55, p=.038, \eta_{\mathrm{p}}{ }^{2}=$
.34 , with a stronger effect of violations after rule-based responses $(\Delta$ $=62 \mathrm{~ms}$ ) compared to after violation responses $(\Delta=23 \mathrm{~ms})$. Finally, the three-way interaction between preceding response type, current response type, and proportion violation was significant, $F(1,11)=13.64$, $p=.004, \eta_{\mathrm{p}}{ }^{2}=.55$, with a significant interaction between preceding and current response type for high-PV blocks, $F(1,11)=8.86, p=.013, \eta_{\mathrm{p}}{ }^{2}$ $=.45$ (see Figure 2, Panel A2), but not for low-PV blocks, $F(1,11)=$ $0.77, p=.399, \eta_{\mathrm{p}}{ }^{2}=.07$ (see Figure 2, Panel A1). None of the remaining effects were significant, $F \leq 2.65, p \geq .132$, for each case.

\section{MOVEMENT TIMES, HIGH-PV-FIRST}

A significant effect of current response type, $F(1,11)=14.61, p=$ $.003, \eta_{\mathrm{p}}{ }^{2}=.57$, was driven by slower responses for violations $(590 \mathrm{~ms})$ than for rule-based behavior ( $555 \mathrm{~ms}$ ). A significant effect of preceding response type, $F(1,11)=5.73, p=.036, \eta_{\mathrm{p}}{ }^{2}=.34$, described responses following rule-based behavior as slower (579 ms) compared to responses following violations (567 ms). Similarly, a significant main effect of proportion violation, $F(1,11)=10.13, p=.009, \eta_{\mathrm{p}}{ }^{2}=.48$, marked responses in the low-PV condition as faster $(550 \mathrm{~ms})$ compared to the high-PV condition (596 ms). The interaction between preceding response type and current response type was significant, $F(1,11)=11.01$, $p=.007, \eta_{\mathrm{p}}{ }^{2}=.50$, with a stronger effect of violations after rule-based responses $(\Delta=59 \mathrm{~ms})$ compared to after violation responses $(\Delta=12$ $\mathrm{ms})$. Finally, the three-way interaction was not significant, $F(1,11)=$ $0.20, p=.665, \eta_{\mathrm{p}}^{2}=.02$, with similar interactions for both, low-PV and high-PV conditions (see Figure 2, Panels B1 and B2). None of the remaining effects were significant, $F \leq 4.35, p \geq .061$, for each case. 


\section{AREAS UNDER THE CURVE, LOW-PV-FIRST}

A significant effect of current response type, $F(1,11)=23.03, p=$ $.001, \eta_{\mathrm{p}}{ }^{2}=.68$, was driven by more contorted responses for violations $\left(39,794 \mathrm{px}^{2}\right)$ than for rule-based behavior $\left(30,245 \mathrm{px}^{2}\right)$. The interaction between preceding response type and current response type was significant, $F(1,11)=9.69, p=.010, \eta_{\mathrm{p}}{ }^{2}=.47$, with a stronger effect of violations after rule-based responses $\left(\Delta=15,323 \mathrm{px}^{2}\right)$ compared to after violation responses $\left(\Delta=3,774 \mathrm{px}^{2}\right)$. Finally, the three-way interaction between preceding response type, current response type, and proportion violation was significant, $F(1,11)=11.27, p=.006, \eta_{\mathrm{p}}{ }^{2}=.51$, with a significant interaction between preceding and current response type for high-PV blocks, $F(1,11)=15.47, p=.002, \eta_{\mathrm{p}}{ }^{2}=.58$ (see Figure 2, Panel C2), but not for low-PV blocks, $F(1,11)=0.16, p=.692, \eta_{\mathrm{p}}{ }^{2}=.02$ (see Figure 2, Panel C1). None of the remaining effects were significant, $F \leq 4.51, p \geq .057$, for each case.

\section{AREAS UNDER THE CURVE, HIGH-PV FIRST}

A significant effect of current response type, $F(1,11)=26.25, p<$ $.001, \eta_{\mathrm{p}}{ }^{2}=.71$, was driven by more contorted responses for violations $\left(31,942 \mathrm{px}^{2}\right)$ than for rule-based behavior $\left(24,112 \mathrm{px}^{2}\right)$. A significant effect of preceding response type, $F(1,11)=9.61, p=.010, \eta_{\mathrm{p}}{ }^{2}=.47$, described responses following rule-based behavior as more contorted $\left(29,942 \mathrm{px}^{2}\right)$ compared to responses following violations $\left(26,112 \mathrm{px}^{2}\right)$. Similarly, a significant main effect of proportion violation, $F(1,11)=$ 5.73, $p=.036, \eta_{\mathrm{p}}{ }^{2}=.34$, marked responses in the low-PV-condition as more contorted $\left(30,811 \mathrm{px}^{2}\right)$ compared to the high-PV-condition $\left(25,243 \mathrm{px}^{2}\right)$. The interaction between preceding response type and current response type was significant, $F(1,11)=23.21, p=.001, \eta_{\mathrm{p}}{ }^{2}$ $=.68$, with a stronger effect of violations after rule-based responses $(\Delta$ $\left.=14,356 \mathrm{px}^{2}\right)$ compared to after violation responses $\left(\Delta=1,297 \mathrm{px}^{2}\right)$. Finally, the three-way interaction was not significant, $F(1,11)=0.39, p$ $=.547, \eta_{\mathrm{p}}{ }^{2}=.03$, with similar interactions for both, low-PV and highPV conditions (see Figure 2, Panels D1 and D2). None of the remaining effects were significant, $F<1, p \geq .951$, in each case.

\section{Discussion}

In Experiment 1, we tested how rule violation performance changes as a function of frequency and recency of rule violations. Participants were confronted with blocks that contained either $25 \%$ or $75 \%$ violation trials, and at first sight, the pattern of results seems rather complex. However, when regarded through the lens of adaptation processes to frequency (in terms of interactions between current response type and proportion violation) and to recency (in terms of interactions between preceding and current response type), the data allows for interesting conclusions. First, the frequency manipulation had no effect on spatial parameters of response execution, while the temporal measure was barely affected, but in a way opposite to what we anticipated. In blocks with a high frequency of violations, temporal response costs for violations increased compared to blocks with a low frequency of violations ${ }^{2}$. This result is difficult to align with the two-step activation model, and we are cautious to read too much into this result before first replicating it (cf. Experiment 2).
Next, we found that the order in which proportions of violations were experienced shaped the way in which violation recency affected responding. Participants who started with a low proportion of violations did not show adaptations to recent violations, replicating our previous results (Wirth, Pfister, Foerster, et al., 2016). However, as soon as these participants encountered a high proportion of violations (in the second half), they did show adaptation to immediately preceding violations. On the other hand, participants who started with a high proportion of violations showed adaptation to recent violations right away, and they continued to do so when proportion of violation dropped. It seems as if for violation tasks, frequency and recency adaptations are not independent mechanisms, but recency adaptations only emerge once a high frequency of violations has been experienced.

One potential factor that might modulate these results further is the sequence of motor responses: In addition to inhibitory mechanisms at the level of task sets (Gade, Souza, Druey, \& Oberauer, 2017; Mayr \& Keele, 2000), research on task switching has also reported systematic inhibitory effects at the level of individual motor responses (Gade, Schuch, Druey, \& Koch, 2014; Grange \& Kowalczyk, 2017; Schuch \& Koch, 2004). However, the data do not support the idea that response sequences influence the results reported above ${ }^{3}$.

On critical reflection, the sample size of Experiment 1 yielded an observed power of .64 for MTs and .65 for AUCs when comparing adaptation patterns across proportion orders (see www.osf.io/a2apv). These results should thus be taken only as preliminary evidence (even though the observed power of the critical three-way interaction of the low-PV-first participants was .92 for MTs and .86 for AUCs). To provide a more robust test for this refinement of the two-step activation model, we set out to replicate these results in Experiment 2, but now doubled the number of participants (resulting in 48 participants in total, 24 per group, which should provide a power $>.80$ for both measures).

\section{EXPERIMENT 2}

\section{Introduction}

Next to replicating the interplay between frequency and recency of violations, we aimed at testing for another factor that might reduce response costs for rule violations: transfer effects that are triggered by a related but separate task. Numerous studies show that conflict effects in one task are reduced when following a different task that recruits executive control, and transfer between separate tasks is strong when they are maximally similar or maximally dissimilar (Notebaert \& Verguts, 2008; for a review, see Braem, Abrahamse, Duthoo, \& Notebaert, 2014).

The reasons for such transfer between tasks are note entirely settled, but they might relate to negative affect, which comes with both interference (Dreisbach \& Fischer, 2012) as well as with rule breaking (Wirth et al., 2017). Negative affect is thought to serve as internal signal which prompts a stronger focus on task-relevant information. Consequently, if subjects experience conflict in a different task, this might result in a stronger focus on task-relevant information such that, 
when subsequently breaking a rule, performance might be less affected by the original mapping rule.

We designed a Simon task that closely resembles the rule task of Experiment 1 (Simon, 1990). In these trials, one of the target areas changed to either red or green, and the color (not the location) indicated if a movement to the left or to the right had to be executed. This resulted in congruent trials (moving towards the colored target area) and incongruent trials (moving away from the colored target area). If conflict adaptation in a Simon task transfers to the rule task, response costs for violations should be smaller after incongruent rather than congruent Simon trials. Of course, this transfer might work the other way round as well, such that recent or frequent rule breaking reduces the cost of spatial incongruency in the Simon task.

\section{Method}

\section{PARTICIPANTS}

A new set of forty-eight participants was recruited $\left(M_{\text {age }}=26.1\right.$ years, $S D=5.3,16$ male, four left-handed) and received either course credit or $8 €$ monetary compensation. All participants gave informed consent, were naïve to the purpose of the experiment, and were debriefed after the session.

\section{APPARATUS, STIMULI AND PROCEDURE}

The experiment was mostly identical to the first experiment. But, intermixed with the rule task, half of the trials now employed a Simon task. In these trials, one of the target areas turned either red or green as soon as they appeared, and participants had to respond to the color by a movement to the left or the right. The location of the colored target area was irrelevant to the task, which resulted in either S-R congruent trials (moving towards the color stimulus) or S-R incongruent trials (moving away from the color stimulus). Again, the S-R mapping for the Simon trials was displayed before a movement started, together with the written instruction "Color" (German: "Farbe"). Simon trials employed 50\% congruent and 50\% incongruent trials throughout the experiment, whereas the rule task trials were still subject to the proportion violation manipulation. All trials within a block were presented in randomized order.

\section{Results}

\section{DATA TREATMENT AND ANALYSES}

The data was treated exactly as in Experiment 1. Accordingly, we again omitted trials in which participants failed to act according to the instruction or failed to hit any of the two target areas at all (4.4\%), trials following an error (3.5\%), and outliers (5.1\%).

We then analyzed each measure separately for each possible trial sequence (rule task $\rightarrow$ rule task, rule task $\rightarrow$ Simon task, Simon task $\rightarrow$ rule task, Simon task $\rightarrow$ Simon task; see Figures 3-6 for descriptive statistics). Analyses for the rule task $\rightarrow$ rule task sequences were performed as in Experiment 1. Again, additional analyses of these sequences, as well as IT results, can be accessed online (www.osf.io/a2apv).

Because all other sequences (rule task $\rightarrow$ Simon task, Simon task $\rightarrow$ rule task, Simon task $\rightarrow$ Simon task) produced less complex data patterns, we report the full $2 \times 2 \times 2 \times 2$ ANOVAs with current response type (rule-based vs. violation for the rule task; congruent vs. incon-

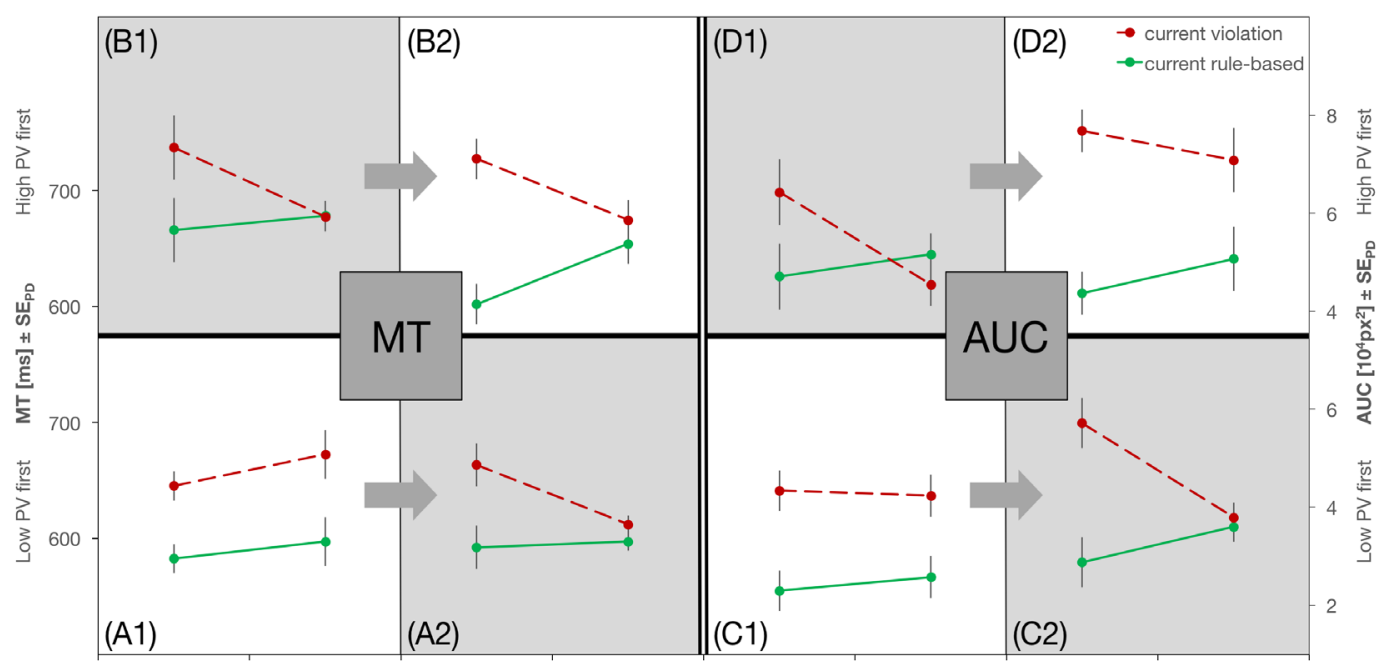

FIGURE 3.

prec. rule-based prec. violation prec. rule-based prec. violation prec. rule-based prec. violation prec. rule-based prec. violation

Results for rule task $\rightarrow$ rule task sequences in Experiment 2. Movement times (MT; left) and areas under the curve (AUC; right) are plotted as a function of preceding response type (abscissa), current response type (continuous green line for rule-based responses; dashed red line for violation responses), and the current proportion of violations (PV; white background for low-PV, gray background for high-PV). Further, the figure is split by proportion order: The lower panels (A and C) represent the low-PVfirst condition, the upper panels (B and D) represent the high-PV-first condition. Note that scaling of the $y$-axes differs between proportion orders. Panels with the number 1 represent the first half of the experiment per proportion order, panels with the number 2 represent the second half. Error bars represent SEs of paired differences, calculated separately for each instance of preceding response type (Pfister \& Janczyk, 2013). 
gruent for the Simon task), preceding response type, and proportion violation (low-PV vs. high-PV) as within-subject factors, and PV order (low-PV-first vs. high-PV-first) as a between-subjects factor for the remaining trial sequences.

\section{RULE TASK $\rightarrow$ RULE TASK SEQUENCES, MOVEMENT TIMES, LOW-PV-FIRST}

A significant effect of current response type, $F(1,23)=30.37, p$ $<.001, \eta_{\mathrm{p}}^{2}=.57$, was driven by slower responses for violations (648 $\mathrm{ms}$ ) than for rule-based behavior (592 ms). The interaction between preceding response type and proportion violation was significant, $F(1$, 23) $=9.87, p=.005, \eta_{\mathrm{p}}{ }^{2}=.30$, with response costs following violations in the low-PV blocks ( $\Delta=21 \mathrm{~ms}$ ) but a response benefit in the high-PV blocks $(\Delta=-23 \mathrm{~ms})$. Finally, the three-way interaction between preceding response type, current response type, and proportion violation was significant, $F(1,23)=4.74, p=.040, \eta_{\mathrm{p}}^{2}=.17$, with a significant interaction between preceding and current response type for high-PV blocks, $F(1,23)=6.76, p=.016, \eta_{\mathrm{p}}^{2}=.23$ (see Figure 3, Panel A2), but not for low-PV blocks, $F(1,23)=0.33, p=.574, \eta_{\mathrm{p}}{ }^{2}=.01$ (see Figure 3, Panel A1). None of the remaining effects were significant, $F \leq 3.33, p$ $\geq .081$, in each case.

\section{RULE TASK $\rightarrow$ RULE TASK SEQUENCES, MOVEMENT TIMES, HIGH-PV-FIRST}

A significant effect of current response type, $F(1,23)=38.24, p<$ $.001, \eta_{\mathrm{p}}{ }^{2}=.62$, was driven by slower responses for violations (704 ms) than for rule-based behavior $(650 \mathrm{~ms})$. A significant effect of preceding response type, $F(1,23)=5.03, p=.035, \eta_{\mathrm{p}}{ }^{2}=.18$, described responses following rule-based behavior as slower $(683 \mathrm{~ms})$ compared to responses following violations $(671 \mathrm{~ms})$. The interaction between current response type and proportion violation was significant, $F(1,23)=4.42$, $p=.047, \eta_{\mathrm{p}}{ }^{2}=.16$, with a smaller effect of violations in high-PV blocks ( $\Delta=35 \mathrm{~ms})$ compared to low-PV blocks $(\Delta=73 \mathrm{~ms})$. Also, the interaction between preceding response type and current response type was significant, $F(1,23)=21.07, p<.001, \eta_{\mathrm{p}}^{2}=.48$, with a stronger effect of violations after rule-based responses $(\Delta=98 \mathrm{~ms})$ compared to after violation responses $(\Delta=10 \mathrm{~ms})$. Finally, the three-way interaction was not significant, $F(1,23)=0.77, p=.390, \eta_{\mathrm{p}}{ }^{2}=.03$, with similar interactions for both low-PV and high-PV conditions (see Figure 3, Panels B1 and B2). None of the remaining effects were significant, $F \leq 2.71, p \geq$ .113 , in each instance.

\section{RULE TASK $\rightarrow$ RULE TASK SEQUENCES, AREAS UNDER THE CURVE, LOW-PV-FIRST}

A significant effect of current response type, $F(1,23)=48.61, p<$ $.001, \eta_{\mathrm{p}}{ }^{2}=.68$, was driven by more contorted responses for violations $\left(45,179 \mathrm{px}^{2}\right)$ than for rule-based behavior $\left(28,359 \mathrm{px}^{2}\right)$. Similarly, a significant main effect of proportion violation, $F(1,23)=5.89, p=.024$, $\eta_{\mathrm{p}}{ }^{2}=.20$, marked responses in the low-PV condition as less contorted $\left(33,595 \mathrm{px}^{2}\right)$ than in the high-PV condition $\left(39,942 \mathrm{px}^{2}\right)$. The interaction between preceding response type and current response type was significant, $F(1,23)=27.87, p<.001, \eta_{\mathrm{p}}{ }^{2}=.55$, with a stronger effect of violations after rule-based responses $\left(\Delta=24,400 \mathrm{px}^{2}\right)$ compared to after violation responses $\left(\Delta=9,241 \mathrm{px}^{2}\right)$. Finally, the three-way interaction

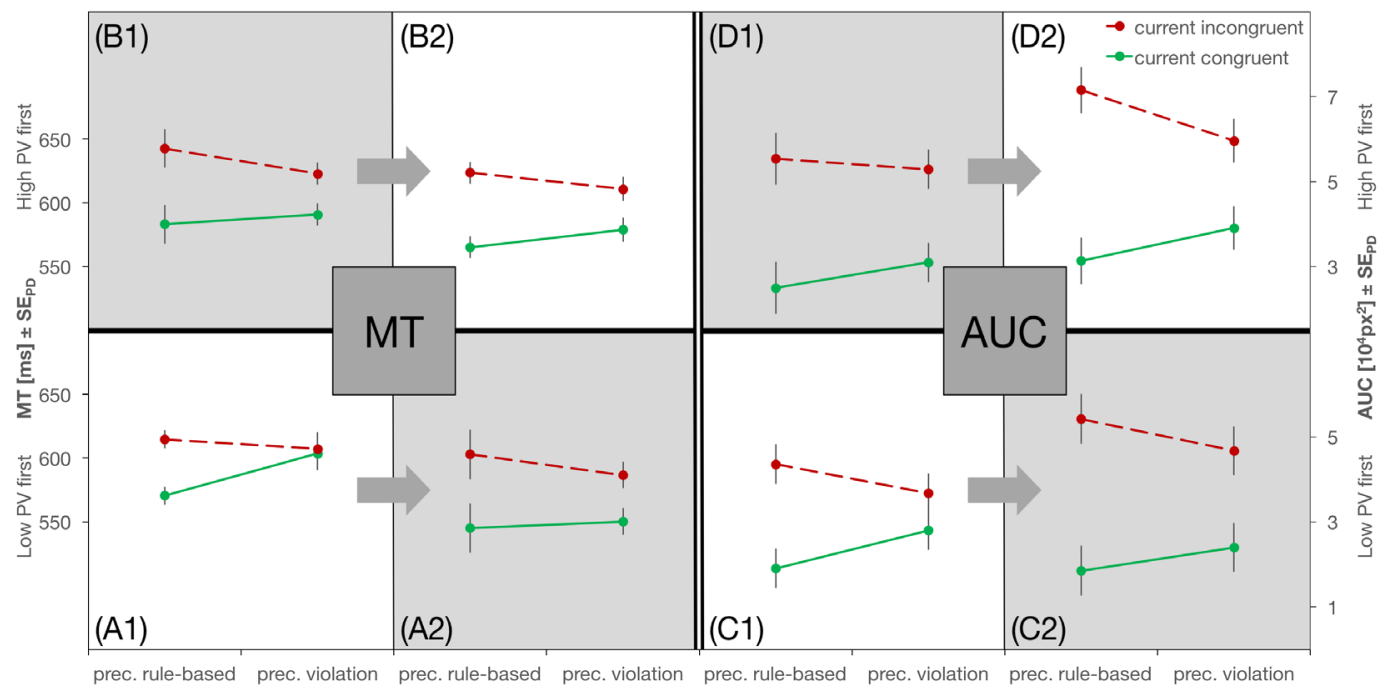

FIGURE 4.

Results for rule task $\rightarrow$ Simon task sequences in Experiment 2. Movement times (MT; left) and areas under the curve (AUC; right) are plotted as a function of preceding response type (abscissa), current response type (continuous green line for congruent; dashed red line for incongruent), and the current proportion of violations (PV; white background for low-PV, gray background for high-PV). Further, the figure is split by proportion order: The lower panels ( $A$ and $C$ ) represent the low-PV-first condition, the upper panels $(B$ and $D$ ) represent the high-PV-first condition. Note that scaling of the $y$-axes differs between proportion orders. Panels with the number 1 represent the first half of the experiment per proportion order, panels with the number 2 represent the second half. Error bars represent SEs of paired differences, calculated separately for each instance of preceding response type (Pfister \& Janczyk, 2013). 
was significant, $F(1,23)=10.14, p=.004, \eta_{p}{ }^{2}=.31$, with a significant interaction between preceding and current response type for high-PV blocks, $F(1,23)=34.26, p<.001, \eta_{p}^{2}=.60$ (see Figure 3, Panel C2), but not for low-PV blocks, $F(1,23)=0.68, p=.417, \eta_{\mathrm{p}}{ }^{2}=.03$ (see Figure 3, Panel C1). None of the remaining effects were significant, $F \leq 3.11, p \geq$ .091 , in each instance.

\section{RULE TASK $\rightarrow$ RULE TASK SEQUENCES, AREAS UNDER THE CURVE, HIGH-PV-FIRST}

A significant effect of current response type, $F(1,23)=24.63, p<$ $.001, \eta_{\mathrm{p}}{ }^{2}=.52$, was driven by more contorted responses for violations $\left(64,269 \mathrm{px}^{2}\right)$ than for rule-based behavior $\left(48,200 \mathrm{px}^{2}\right)$. Similarly, a significant main effect of proportion violation, $F(1,23)=5.10, p=.034$, $\eta_{\mathrm{p}}{ }^{2}=.18$, marked responses in the low-PV condition as more contorted $\left(60,426 \mathrm{px}^{2}\right)$ compared to the high-PV condition $\left(52,044 \mathrm{px}^{2}\right)$. There was an interaction between current response type and proportion violation, $F(1,23)=13.54, p=.001, \eta_{\mathrm{p}}{ }^{2}=.37$, with a larger violation effect in the low-PV condition $\left(\Delta=26,626 \mathrm{px}^{2}\right)$ compared to the high-PV condition $\left(\Delta=5,512 \mathrm{px}^{2}\right)$. The interaction between preceding response type and current response type was significant, $F(1,23)=12.08, p=$ $.002, \eta_{\mathrm{p}}^{2}=.34$, with a stronger effect of violations after rule-based responses $\left(\Delta=25,138 \mathrm{px}^{2}\right)$ compared to after violation responses $(\Delta=$ $\left.7,000 \mathrm{px}^{2}\right)$. Finally, the three-way interaction was not significant, $F(1$, 23) $=1.15, p=.295, \eta_{\mathrm{p}}^{2}=.05$, with similar interactions for both low-PV and high-PV conditions (see Figure 3, Panels D1 and D2). None of the remaining effects were significant, $F \leq 4.28, p \geq .050$, in each case.

\section{RULE TASK $\rightarrow$ SIMON TASK SEQUENCES, MOVEMENT TIMES}

A significant effect of current response type, $F(1,46)=53.78, p<$ $.001, \eta_{\mathrm{p}}{ }^{2}=.53$, was driven by faster responses for congruent ( $573 \mathrm{~ms}$ ) than for incongruent responses $(614 \mathrm{~ms})$. Also, there was an interaction between current response type and preceding response type, $F(1$, $46)=14.27, p<.001, \eta_{\mathrm{p}}^{2}=.24$, with a stronger congruency effect after rule-based responses $(\Delta=55 \mathrm{~ms})$ than after violation responses $(\Delta=$ $26 \mathrm{~ms}$ ). This interaction held true for all combinations of proportion violation and proportion order (see Figure 4, A and B), as indicated by all higher-order interactions including both factors returning nonsignificant results, $F<1, p \geq .451$, for all combinations. None of the remaining effects were significant, $F \leq 3.56, p \geq .066$, in each case.

\section{RULE TASK $\rightarrow$ SIMON TASK SEQUENCES, AREAS UNDER THE CURVE.}

A significant effect of current response type, $F(1,46)=69.18, p$ $<.001, \eta_{\mathrm{p}}^{2}=.60$, was driven by more direct responses for congruent $\left(26,996 \mathrm{px}^{2}\right)$ than for incongruent trials $\left(52,593 \mathrm{px}^{2}\right)$. Proportion order interacted with proportion violation, $F(1,46)=9.62, p=.003, \eta_{p}^{2}=.17$, with benefits in low-PV blocks for participants who started with the low-PV condition $\left(\Delta=4,005 \mathrm{px}^{2}\right)$ but costs for those who started with the high-PV condition $\left(\Delta=-9,322 \mathrm{px}^{2}\right)$. Also, there was an interaction between current response type and preceding response type, $F(1$, $46)=31.22, p<.001, \eta_{\mathrm{p}}{ }^{2}=.40$, with a stronger congruency effect after rule-based responses $\left(\Delta=32,702 \mathrm{px}^{2}\right)$ than after violation responses

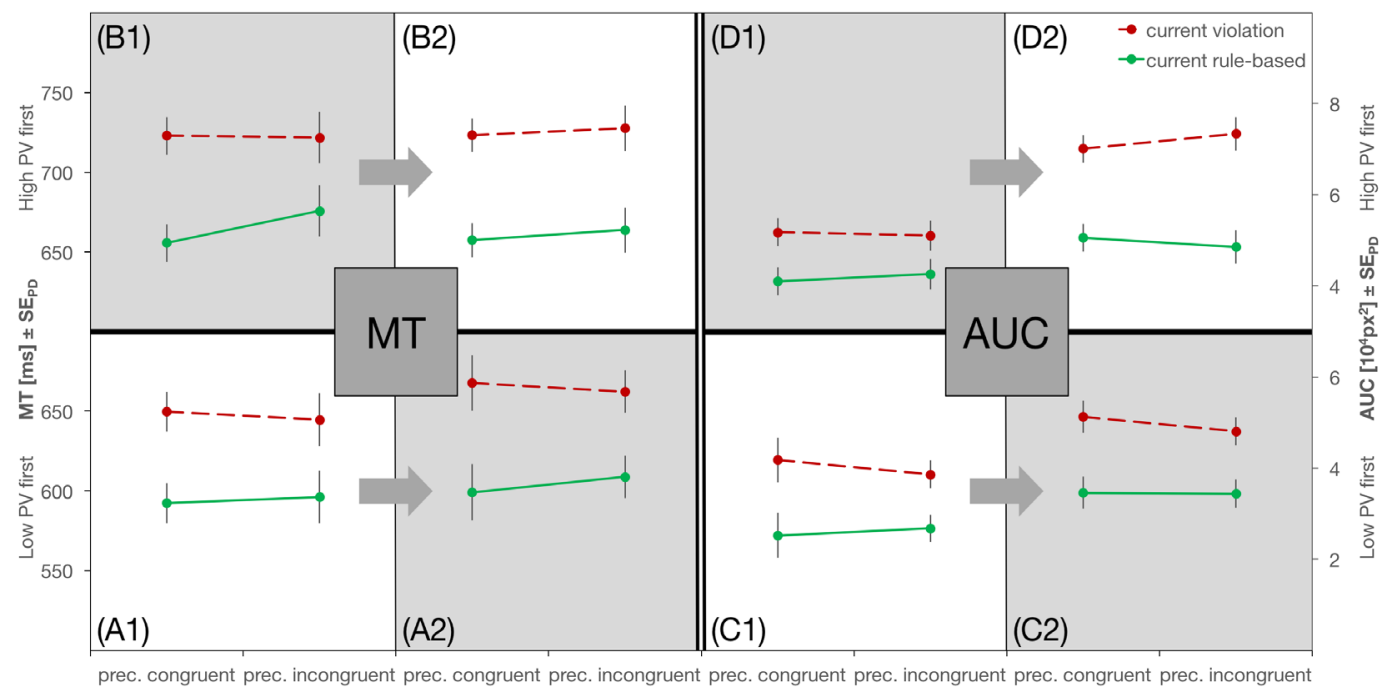

FIGURE 5.

Results for Simon task $\rightarrow$ rule task sequences in Experiment 2. Movement times (MT; left) and areas under the curve (AUC; right) are plotted as a function of preceding response type (abscissa), current response type (continuous green line for rule-based responses; dashed red line for violation responses), and the current proportion of violations (PV; white background for low-PV, gray background for high-PV). Further, the figure is split by proportion order: The lower panels ( $\mathrm{A}$ and $\mathrm{C}$ ) represent the low-PVfirst condition, the upper panels (B and D) represent the high-PV-first condition. Note that scaling of the $y$-axes differs between proportion orders. Panels with the number 1 represent the first half of the experiment per proportion order, panels with the number 2 represent the second half. Error bars represent SEs of paired differences, calculated separately for each instance of preceding response type (Pfister \& Janczyk, 2013). 
$\left(\Delta=18,492 \mathrm{px}^{2}\right)$. This interaction held true for all combinations of proportion violation and proportion order (see Figure 4, C and D), $F \leq$ $1.21, p \geq .278$, for all combinations. None of the remaining effects were significant, $F \leq 2.12, p \geq .149$, in each case.

\section{SIMON TASK $\rightarrow$ RULE TASK SEQUENCES, MOVEMENT TIMES}

A significant effect of current response type, $F(1,46)=73.26, p<$ $.001, \eta_{\mathrm{p}}^{2}=.61$, was driven by faster responses for rule-based $(631 \mathrm{~ms})$ than for violation responses $(690 \mathrm{~ms})$. The interaction between current response type and preceding response type was not significant, $F(1$, 46) $=1.97, p=.167, \eta_{\mathrm{p}}^{2}=.04$, and this held true for all combinations of proportion violation and proportion order (see Figure 5, Panels A and B), $F<1, p \geq .460$, for all combinations. None of the remaining effects were significant, $F \leq 1.06, p \geq .309$, for all instances.

\section{SIMON TASK $\rightarrow$ RULE TASK SEQUENCES, AREAS UNDER THE CURVE}

A significant effect of current response type, $F(1,46)=69.32, p$ $<.001, \eta_{\mathrm{p}}^{2}=.60$, was driven by more direct responses for rule-based $\left(37,959 \mathrm{px}^{2}\right)$ than for violation trials $\left(53,276 \mathrm{px}^{2}\right)$. Proportion order interacted with proportion violation, $F(1,46)=21.91, p<.001, \eta_{\mathrm{p}}^{2}=.32$, with benefits in low-PV blocks for participants who started with the low-PV condition $\left(\Delta=8,990 \mathrm{px}^{2}\right)$ but costs for those who started with the high-PV condition $\left(\Delta=-14,054 \mathrm{px}^{2}\right)$. Also, there was an interaction between current response type and proportion violation, $F(1,46)$ $=8.26, p=.006, \eta_{\mathrm{p}}{ }^{2}=.15$, with a stronger violation effect in low-PV blocks $\left(\Delta=18,212 \mathrm{px}^{2}\right)$ compared to high-PV blocks $\left(\Delta=12,422 \mathrm{px}^{2}\right)$. The interaction between current response type and preceding response type was not significant, $F(1,46)=0.34, p=.562, \eta_{\mathrm{p}}{ }^{2}=.01$, and this held true for all combinations of proportion violation and proportion order (see Figure 5, C and D), $F \leq 1.90, p \geq .174$, for all combinations. None of the remaining effects were significant, $F \leq 1.43, p \geq .237$, for all cases.

\section{SIMON TASK $\rightarrow$ SIMON TASK SEQUENCES, MOVEMENT TIMES.}

A significant effect of current response type, $F(1,46)=66.03, p<$ $.001, \eta_{\mathrm{p}}{ }^{2}=.59$, was driven by faster responses for congruent (556 ms) than for incongruent responses $(593 \mathrm{~ms})$. Also, there was an interaction between current response type and preceding response type, $F(1$, $46)=43.90, p<.001, \eta_{\mathrm{p}}^{2}=.49$, with a stronger congruency effect after congruent responses $(\Delta=52 \mathrm{~ms})$ than after incongruent responses ( $\Delta$ $=22 \mathrm{~ms}$ ). This interaction held true for all combinations of proportion violation and proportion order (see Figure 6, Panels A and B), F $<1, p \geq .421$, for all combinations. None of the remaining effects were significant, $F \leq 3.34, p \geq .074$, for all cases.

\section{SIMON TASK $\rightarrow$ SIMON TASK SEQUENCES, AREAS UNDER THE CURVE}

A significant effect of current response type, $F(1,46)=66.38, p$ $<.001, \eta_{\mathrm{p}}^{2}=.59$, was driven by more direct responses for congruent $\left(22,666 \mathrm{px}^{2}\right)$ than for incongruent trials $\left(46,194 \mathrm{px}^{2}\right)$. Also, there was a significant effect of preceding response type, $F(1,46)=24.04, p<.001$, $\eta_{\mathrm{p}}^{2}=.34$, with faster responses after incongruent $\left(32,232 \mathrm{px}^{2}\right)$ compared

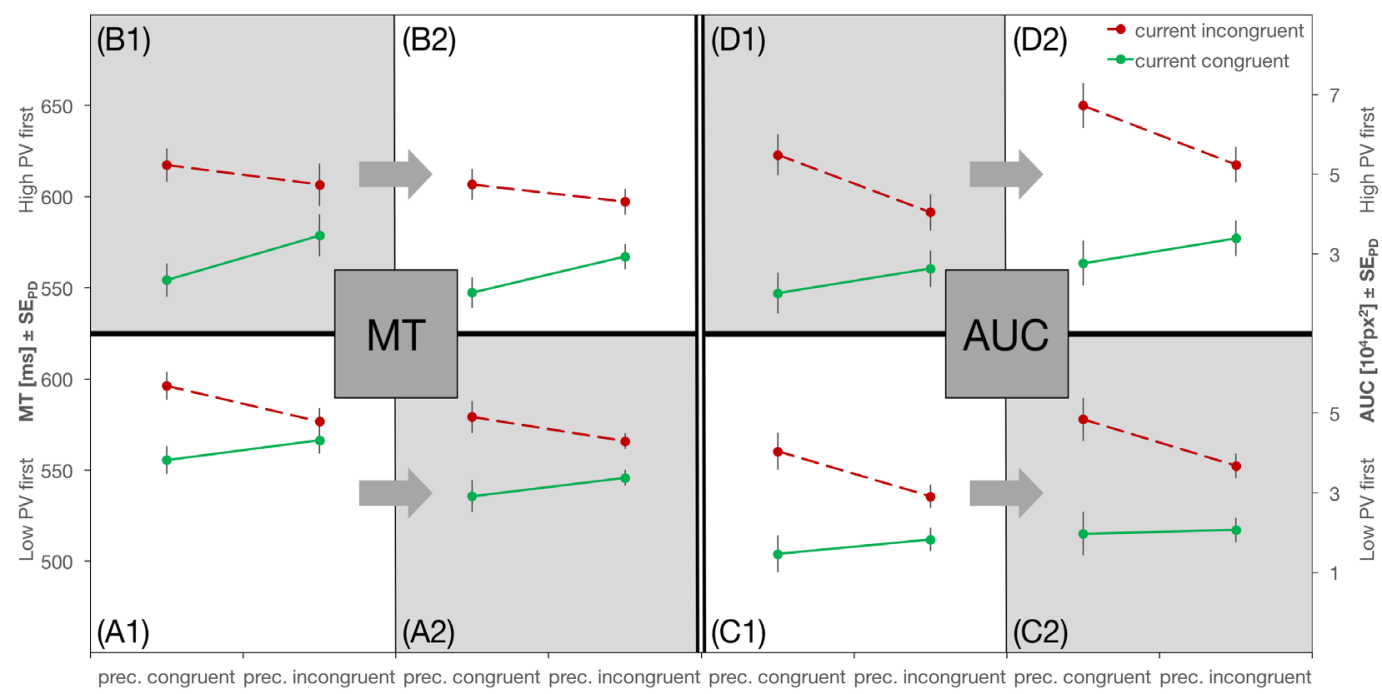

FIGURE 6.

Results for Simon task $\rightarrow$ Simon task sequences in Experiment 2. Movement times (MT; left) and areas under the curve (AUC; right) are plotted as a function of preceding response type (abscissa), current response type (continuous green line for congruent; dashed red line for incongruent), and the current proportion of violations (PV; white background for low-PV, gray background for high-PV). Further, the figure is split by proportion order: The lower panels (A and C) represent the low-PV-first condition, the upper panels ( $B$ and $D$ ) represent the high-PV-first condition. Note that scaling of the $y$-axes differs between proportion orders. Panels with the number 1 represent the first half of the experiment per proportion order, panels with the number 2 represent the second half. Error bars represent SEs of paired differences, calculated separately for each instance of preceding response type (Pfister \& Janczyk, 2013). 
to after congruent responses $\left(36,628 \mathrm{px}^{2}\right)$. Proportion order interacted with proportion violation, $F(1,46)=18.75, p<.001, \eta_{\mathrm{p}}{ }^{2}=.29$, with benefits in low-PV blocks for participants who started with the low-PV condition $\left(\Delta=5,777 \mathrm{px}^{2}\right)$, but with costs for those who started with the high-PV condition $\left(\Delta=-9,866 \mathrm{px}^{2}\right)$. Also, there was an interaction between current response type and preceding response type, $F(1$, 46) $=61.05, p<.001, \eta_{\mathrm{p}}{ }^{2}=.57$, with a stronger congruency effect after congruent responses $\left(\Delta=32,191 \mathrm{px}^{2}\right)$ than after incongruent responses $\left(\Delta=14,864 \mathrm{px}^{2}\right)$. This interaction held true for all combinations of proportion violation and proportion order (see Figure 6, Panels C and D), $F \leq 2.56, p \geq .0116$, for all combinations. None of the remaining effects were significant, $F \leq 1.28, p \geq .264$, for all cases.

\section{Discussion}

In Experiment 2, our first aim was to replicate the results of Experiment 1. With increased power, we had a look at the data pattern that again emerged in rule task $\rightarrow$ rule task sequences. Now, the frequency manipulation emerged as expected ${ }^{4}$. In all cases, however, violations came with notable costs even if rule violations were more frequent than rule-based responses, even in blocks with rule violations three times as frequent as rule-based responses, the data pattern did not reverse. And again, recency adaptations only emerged when a high frequency of violations had already been experienced. This strongly suggests that frequency and recency adaptations are not independent mechanisms in the rule task, but that recency adaptations only occur if a violation task set is or has been used with sufficient frequency. On the other hand, frequency adaptations do not seem to depend on recency, as frequency manipulations even emerged when no recency adaptations from a directly preceding rule violation were possible (e.g., visible in the AUC data in Simon task $\rightarrow$ rule task sequences).

Our next goal for Experiment 2 was to test whether transfer effects from a separate task could modulate the response costs for violations. To do so, we designed a Simon task that closely resembled the rule task. The data is much less complex here: After a violation, responses to incongruent Simon trials are facilitated compared to after a rule-based response (in rule task $\rightarrow$ Simon task sequences). This transfer further highlights that rule violations indeed entail cognitive conflict between the rule-based and the violation response: Up to now, the only direct evidence supporting this idea was the trajectory deviations towards the rule-based response option during rule violations (which we also show here). However, this result could also be obtained assuming that violations are simply more complex and demanding, with more difficult responses producing less direct trajectories. Albeit being less parsimonious, such a model would produce similar spatial effects without assuming any conflict. An absent effect of rule violations on the frontocentral N2 component could further be taken as evidence against cognitive conflict during rule violations (Pfister, Wirth, Schwarz, Foerster, et al., 2016). The present transfer from the rule task to the Simon task, however, clearly speaks in favor of the notion of cognitive conflict:
Having violated a rule in a previous trial reduces conflict effects in the tried-and-tested Simon conflict task, suggesting that violations indeed entail cognitive conflict, and experiencing these conflicts can consequently reduce conflict effects in a subsequent Simon task.

And even though the Simon task in principle produced the wellknown within-task adaptation effects (in Simon task $\rightarrow$ Simon task sequences, as a manipulation check), these adaptation effects do not transfer to the rule task (in Simon task $\rightarrow$ rule task sequences). So the transfer between the tasks is asymmetric, such that only violations seem to affect subsequent Simon responses, and not the other way round.

One might wonder what exactly transfers between the two tasks. Even though both tasks were designed to share a maximum of features, which should make transfer more likely (Braem et al., 2014), the cognitive processes that they require strongly differ. Incongruent Simon trials require the translation of relevant perceptual information (color) into a motor response while shielding this process from the taskirrelevant location of the stimulus. Here, the relevant features have to be activated while simultaneously inhibiting the irrelevant features. In comparison, violations are thought to entail a dual-activation of the rule-based and the violation task set, with an inhibition of the violation task set afterwards to proactively reduce task set competition in the next trial. It might be that the inhibition after a violation can improve the subsequent inhibition of task-irrelevant features in a Simon task, but the inhibition that is exercised during a Simon task cannot figure as a benefit during a rule violation, as producing a violation response does not entail an inhibition. Only after the violation response has been completed, an inhibition process is required, but at that point, a transfer benefit can no longer emerge. Further, the transfer asymmetry could be explained by assuming that rule violations require two processes to allow for a response selection: an inhibition and a modulation (e.g., negation of the original rule), while incongruent Simon trials require only an inhibition process to arrive at the correct response. So after a violation, an inhibition process has already been recruited and performance in a subsequent incongruent Simon trial can improve, but after an incongruent Simon trial, no modulation process is at work, which would be required to violate a rule.

However, it might not be the violation task itself that causes the adaptation in the Simon task, but rather the affect that comes with violating a rule (Wirth et al., 2017). Violations have been shown to entail a negative affective component, and adaptation effects emerge especially in negative settings (van Steenbergen, Band, \& Hommel, 2009, 2010; Wirth, Pfister, \& Kunde, 2016). This could also explain why, for participants that start with the low-PV condition, there is no benefit after a violation for a subsequent violation (see Figure 3, Panel A1), but for a subsequent Simon task (see Figure 5, Panel A1). If not the violation task itself causes the adaptation, but the affective signal that it triggers, this could explain why the Simon task, which has been demonstrated to respond to mood manipulations, shows an adaptation effect after a violation, but a subsequent violation that might be more robust towards modulations by affect, does not. 


\section{GENERAL DISCUSSION}

Rule violations are difficult to plan and execute (Pfister, Wirth, Schwarz, Steinhauser, \& Kunde, 2016), but some individuals suffer less from the burdens of nonconformity than others (Jusyte et al., 2017). How can we facilitate rule breaking within one and the same person? With the current experiments, we tested whether the difficulty to break rules can be overcome or at least reduced. To do so, we varied the ratio of required rule-based responses and rule violations in Experiment 1 to test for reductions of rule violation costs as a function of violation frequency and recency. Experiment 2 additionally tested whether conflict in an unrelated task can help to overcome rule violation costs.

\section{Summary of the Results}

To sum up the results, we first replicated that it is indeed harder to execute a rule violation, with significant temporal and spatial response costs relative to rule-based behavior. This was true for blocks that employ a low proportion of violations (and which by itself might be explained by the infrequent presentation of these trials), but crucially this was also the case with the majority of trials requiring rule violations in a block. If just the relative required frequency of both responses determined their ease of execution, then we should find that with a high proportion of violations, violations should become easier to execute than rule-based responses. However, the direction of this difference never reversed and rule violations were always slower and more contorted than rule-based responses, suggesting that violations indeed entail a cognitive detour with an initial activation of the original rule, as described in the two-step activation model (Wirth, Pfister, Foerster, et al., 2016).

When it comes to the proportion of rule violations, we find that there is no systematic influence on response execution. The difficulty to execute rule violations did not even become slightly easier with more violations required (see Footnote ${ }^{4}$ ), which in itself is interesting, as this influence is usually observed in conflict tasks (Logan \& Zbrodoff, 1979). Again, this result is compatible with the two-step activation model, assuming that every single violation requires the described cognitive detour. Even when it is required often, the cognitive detour itself does not change. Recency adaptations, however, showed an interesting and remarkably stable pattern of results. In short, recency adaptations (in terms of lower violation costs if a violation had just been performed relative to if a rule-based response had just been performed) emerged only when a high proportion of violations is currently present or has already been experienced. If participants started with a low proportion of violations, they showed no recency benefit (replicating Wirth, Pfister, Foerster, et al., 2016). Although frequency and recency are usually described as being independent (Torres-Quesada et al., 2013), in the rule task they seemed to work in concert.

Finally, the introduction of the Simon task in Experiment 2 produced asymmetric transfer effects between the two tasks. While a prior violation reduced incongruency effects in the next trial, prior incongruency did not modulate the response parameters in the rule task. This asymmetry might be driven by negative affect that is involved in both tasks (see the Discussion section of Experiment 2).

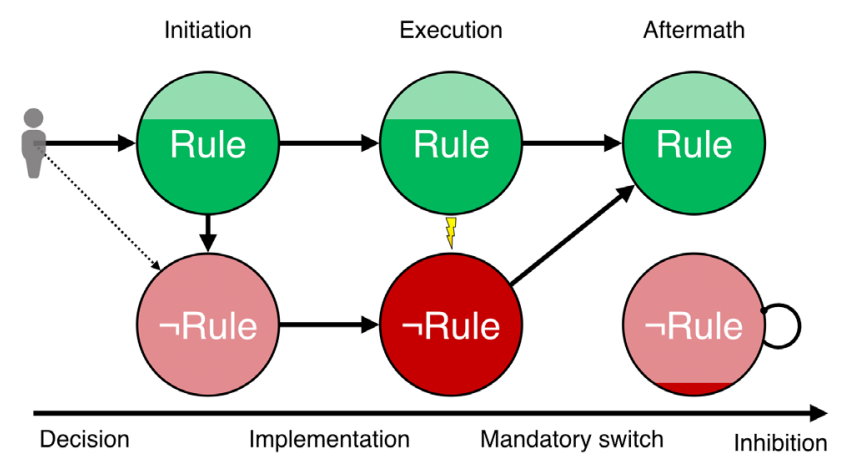

FIGURE 7.

The Decision - Implementation - Mandatory switch - Inhibition (DIMI) model. Following the arrows, the model describes the two routes for following or breaking a rule. The circles represent the task sets for following or breaking a rule, the level within the circles depicts the degree of implementation of each task set. First, participants choose a task set to follow or break a rule. In this step, either the preimplemented task set for rule-based responses is chosen, or a new task set for a violation response is created (by means of inversion, negation, or transformation). There is a clear hierarchy between these two task sets, with the lower level (red circles) strongly depending on the upper level (green circles) for its meaning. Next, the chosen task set has to be fully implemented to allow for the selection of a response. While the task set for rule-based responses can stand on its own, the violation task set is only represented as a transformation of the rule-based task set and, therefore, cannot be active alone. Breaking a rule thereby inherently creates conflict between both task sets. To avoid this conflict in the next trials, one of the task sets has to be inhibited. But as the violation task set cannot be entertained on its own, a mandatory switch back to the rulebased task set is required, and the violation task set is actively inhibited afterwards. An animation that illustrates rule-based and violation responses (including sequential modulations) according to this model is provided online (www.osf.io/a2apv). 


\section{The Two-step Activation Model Revisited}

These last two results, the interplay of frequency and recency and the asymmetric transfer with another conflict task, cannot be explained by the two-step activation model. Therefore, we want to discuss an evolved version of the two-step activation model. We label this model the Decision-Implementation-Mandatory switch-Inhibition (DIMI) model (see Figure 7).

The DIMI model makes the following assumptions (all taken from the original two-step activation model):

- Rule-based and violation responses rely on two distinct task sets. The violation task set does not stand on its own, though, but it is construed of the original rule plus a modulator that alienates its meaning (Wirth, Pfister, Foerster, et al., 2016). There is a hierarchical relationship between the two task sets, so that violations (on the lower level, red circles) require that the original rule (the upper level, green circles) is still accessible.

- Choosing one or the other task set typically takes place before response initiation. Implementation, by contrast, is not necessarily completed before response initiation and can continue even during response execution (Scherbaum et al., 2010).

- Humans are generally prepared to abide by the rules, so the task set for rule-based behavior can be construed as the default (Asch, 1956; Milgram, 1963). Therefore, the task set for rule-based responding is partially pre-implemented.

- The simultaneous implementation of two task sets causes interference (Hsieh et al., 2012; Meiran et al., 2010).

Let us first consider the case of rule-consistent behavior. Choosing the preimplemented rule-based task set is relatively effortless, so responses can be initiated quickly. The implementation of this task set is easier and faster than when violating rules, therefore rule-based responses are completed faster. After its use, the strength of implementation levels off to its initial state over time.

Let us now consider rule violations: If one decides to break a rule, the task set for rule violations first has to be created by modulating the original rule-based task set. This modulation can consist of any operator that alienates the meaning of the original task set (in our case, participants probably used a negation), which ultimately creates a new, dependent task set for rule violations. Dependent here means that the task set is represented as a combination of the original task set plus the modulating operator ("with strings attached" to the original rule). This process takes some time. Consequently, violation responses are initiated comparably slow. This new task set now has to be implemented to allow for response selection, but as it has only just been derived, its implementation takes far longer. Simultaneously, the original rule on the upper hierarchy level must be active so that its content can be accessed. This dual implementation during rule violations could explain the persisting influence of the original rule (Pfister, Wirth, Schwarz, Steinhauser, \& Kunde, 2016). However, implementing two task sets at once is difficult and might lead to interference (Hsieh et al., 2012; Kuhns et al., 2007; Meiran et al., 2010), so one of the task sets is best inhibited before the next trial. But as the violation task set cannot stand on its own (the lower hierarchy level depends on the upper level), it is the violation task set that is actively inhibited after use, and a mandatory task switch back to the rule-based task set is triggered after violating a rule. Hence, violating the rules cannot become the default (Hoffman, 1981). And as the violation task set is only required rarely, it is inhibited strongly.

So far, this is a redescription of the original model, and it accounts for the behavioral signature of rule violations within a trial, as well as for sequential effects. For a subsequent rule violation, the decision process (which occurs during ITs) would benefit from a recent violation, as the violation task set would not have to be derived anew. Instead, choosing between following or breaking the rule would follow a general task switching logic: Repeating the currently active task set (which, after the mandatory switch is rule-based responding) would be easier than switching to the currently inhibited task set (represented by the dotted arrow in Figure 7). However, if the violation task set had not been implemented for a longer period of time, it is deallocated to further reduce competition between the two task sets. In this case, the decision to follow a rule would again be very fast, and violating a rule would again entail the derivation process, which would be very slow. This modeled pattern of results is actually backed up by empirical data that shows that the decision to follow or break a rule (reflected by ITs) produces sequential adaptation effects, with large costs for violations after a rule-based response, and smaller costs for violations after a violation response (see online material, www.osf.io/a2apv; Wirth, Pfister, Foerster, et al., 2016).

However, when it comes to the actual execution of the response, the model predicts no repetition benefits for violations: As every trial includes a mandatory switch back to the rule-based task set and an inhibition of the violation task set, choosing the inhibited task set becomes faster, but it has to be implemented anew as if it had not been used before. The implementation process takes longer for violations than for the preimplemented rule-based task set (reflected by MTs), and the inhibition process afterwards annuls any chance for residual activation of the task set to improve a subsequent violation. Also, the relative degree of implementation of the competing task set allows for predictions of the spatial attraction towards the alternative response (reflected by AUCs). Again, these predictions are reinforced by the empirical data that suggests that rule violation trajectories are heavily attracted to the rule based target location and that neither the temporal nor spatial measures of the response execution are the subject to sequential modulation (when high frequency of violations has not been experienced yet).

\section{Addressing Frequency Within the Model}

Our current results show that this is only true for participants that start with a low proportion of violations (which probably is the most externally valid scenario, see Figure 2, Panel A1 and Figure 3, Panel A1). The frequency manipulation that we introduced in Experiments 1 and 2 still has to be addressed in the model. To account for this within the model, the following assumptions were added: 
- The proportion of violations is proportional to the self-inhibition of the violation task set after use, the more it is required, the less it is inhibited.

- Once participants have attenuated the self-inhibition process, the strength of inhibition is fixed, and even with a later low proportion of violations, the self-inhibition is not enlarged (which might reflect a strategic trade-off).

With a high frequency of violations, the violation task set is required more often, and consequently, it is inhibited less strongly after use. The inhibition process is attenuated to facilitate a likely subsequent violation that could now benefit from residual activity from the previous trial. This marks a trade-off: Residual activation in the violation task set improves a subsequent violation but increases the chance of interference between the two task sets. Thus, a subsequent rule-based response should be more difficult. Taken together, this model predicts that with a high proportion of violations, ITs should produce a smaller violation effect, because both task sets are constantly implemented to a certain degree. Also, the execution parameters should now produce smaller violation effects, and even adaptation effects, as repeated violations can benefit from residual activation from the previous trial, which should improve the time of implementation (reflected by shorter times to complete a violation, MTs), and the competing influence of the rulebased task set during violations should lessen with less use, allowing for more efficient spatial responses (smaller AUCs).

Crucially, after a violation, there is still a mandatory switch back to the rule-based task set. This allows for the odd prediction that even with a high proportion of violations, the infrequent rule-based responses should still be faster and more efficient than frequent violations (again stressing that violations cannot become our default). And again, all these predictions are met by the empirical data presented in this article. Violation effects slightly diminish with a higher frequency (but see Footnote ${ }^{3}$ ), still, violations never become faster or more efficient than rule-based responses, even for response execution sequential modulations now emerged. However, while this shows that recency adaptations strongly depend on the factor frequency (at least for the response execution), frequency has an effect even when recency cannot be involved: In Experiment 2, when switching from a Simon to a rule task, recency adaptations could not emerge, but frequency still modulated the results, which can be explained by the attenuated inhibition process in the condition with a high proportion of violations.

When it comes to transfer within the rule task, we might still wonder what preconditions have to be met so that transfer from one trial to the next occurs (cf. Braem et al., 2014). The rules that we used so far are simple stimulus-response rules (if target $\mathrm{X}$, then left response, if target $\mathrm{Y}$, then right response). But rules could also be more abstract, as, for example, with semantic categorization rules that are valid for a multitude of stimuli (if stimulus is male, then left response, if stimulus is female, then right response). Currently, it is unclear whether the results that we report here require rules that operate on a concrete level (S-R rules) or whether they can also be more abstract (semantic level), or whether transfer could even be found between two separate rules (Badre, 2008; Badre \& Wagner, 2006). It seems plausible that the mechanisms do indeed operate also at the semantic level, given that such sematic rules are readily retrieved and exert a particularly strong impact decision making and behavior (Dreisbach, 2012; Dreisbach, Goschke, \& Haider, 2007). The current experiments were not designed to answer these questions, however, and future research could address them by employing a categorization rule with a broad spectrum of stimuli, or even two exclusive rules.

Further, we could assume that there is also an influence of trials that not only directly precede a response (trial $n-1$ influence), but exceed this timeframe (e.g., $n-2$ or $n-3$ influence). Recently, it has been shown that with a high proportion of conflict, even more distant trials can influence processing on the current trial (Aben, Verguts, \& Van den Bussche, 2017). Therefore, especially in the high-PV condition that shows a stable recency influence, we might find influences of trials that lie even further back in time. However, the current experiments were not designed to illustrate such an influence, and a thorough analysis of influences beyond the previous trial is not possible due to a small number of data points per cell. If such an influence were found, this would underline the idea that the processing of rule violations and the processing of cognitive conflict share common mechanisms.

\section{Explaining Transfer Effects Within the Model}

What is now left to explain within the DIMI model are the transfer effects between the rule task and the Simon task. The observed transfer effects were asymmetrical. Only after a violation was a response to an incongruent Simon stimulus facilitated, but after an incongruent Simon trial, no adaptation effects emerged in the rule task. This asymmetry might be driven by an affective account that has already been discussed (Dreisbach \& Fischer, 2012; see the Discussion section of Experiment 2), but can also be accommodated in the presented model.

First, let us summarize the processing steps that are assumed for completing a Simon task. With stimulus onset, the stimulus' location and color can be processed. The color always indicates the required spatial response. However, extracting this information is not automatic. By contrast, extracting the required response from the location is easy and relatively automatic. In a congruent trial, both features hold the same information, so both features are considered in response selection to arrive at a fast decision. In an incongruent trial, this strategy would be detrimental because the location of the stimulus provokes an error. Here, the location has to be inhibited to give way for the processing of the stimulus color. In the next trial, this inhibition can be maintained so that response selection in congruent trials is slower, but response selection in incongruent trials is now less affected by the stimulus location.

A rule violation equally requires that two response tendencies are resolved, the automatic, rule-based tendency versus the currently required violation response. As described earlier, the cognitive system has to ensure that the violation tendency is put into action, but it cannot inhibit the representation of the original rule to do so. The violation task set operates on a lower level of the hierarchy and is only represented as a transformation of the original rule and-without the original rule in 
mind-has no meaning of its own. Therefore, an inhibition is required only after the response has been selected and executed, to minimize the chance of interference in the next trial.

Overall, both the Simon and the rule task require the inhibition of information that may cause interference. However, the point in time at which this inhibition is required differs. That might explain why the transfer between the two tasks is asymmetrical. After a rule violation, there is an inhibition process, which might enable the suppression of the irrelevant location of the stimulus in the Simon task more efficiently. In an incongruent trial, there is also an inhibition process, but it cannot be transferred to the rule task, as the rule task only employs an inhibition process after all is set and done. There might be transfer from the Simon task to the inhibition at the end of a violation, but if this was the case, it cannot be measured by parameters that emerge during a violation.

\section{CONCLUSION}

How, then, can we reduce the burdens that come with nonconformity? This is how to be a rule breaker: Do it often, and then do it repeatedly. Having violated a rule recently only improves the planning of a further violation but the execution is still heavily crippled. And while training alone diminished the response costs for violation, the greatest benefit results from combining both training and accessibility. Accessibility to a task that presumably resembles the rule violation does not help. However, training to break one rule might transfer to the violation of a second rule. With two rules that have to be broken, both tasks require similar operations (modulation and inhibition) that might allow for transfer. These questions still have to be addressed in further research. For now, the best advice to violate a rule efficiently is to keep the corresponding task set implemented as strongly as possible, and that can best be done by using is frequently and having it used recently.

\section{DISCLAIMER}

The authors take no responsibility for the consequences of any actions that may occur while employing the advice given above.

\section{FOOTNOTES}

${ }^{1}$ For a discussion on whether rule violations produce conflict that requires cognitive control at all, see the Discussion section of Experiment 2.

${ }^{2}$ This interaction was only statistically significant for the low-PVfirst group. Post-hoc Bayesian analyses show that there is overall only weak evidence for an interaction between current response type and proportion violation in $\mathrm{MTs}, \mathrm{BF}_{01}=0.27$.

${ }^{3}$ When analyzing the data including the factor response sequence (repetition vs. switch), we found a main effect of response sequence for MTs, $F(1,22)=14.98, p=.001, \eta_{\mathrm{p}}^{2}=.41$, with faster response execution for repetitions $(619 \mathrm{~ms})$ than for switches $(604 \mathrm{~ms})$. However, there was no interaction including the factor response sequence, $F \leq 1.97$, $p \geq .173$, in each instance. For AUCs, there was neither a significant main effect nor a significant interaction including the factor response sequence, $F \leq 2.24, p \geq .149$, in each instance. Overall, this suggests that not the executed motor response (move finger left vs. right) is relevant for sequential adaptation, but the cognitive process that was required to select the movement.

${ }^{4}$ Again, Bayesian analyses suggest that there is barely any evidence in favor of the interaction between current response type and proportion violation in $\mathrm{MTs}, \mathrm{BF}_{01}=0.86$, and evidence against its existence in AUCs, $\mathrm{BF}_{10}=25.96$. Overall, this suggests that in both experiments, these interactions, although at times significant, are fragile and should not be interpreted any further.

\section{REFERENCES}

Aben, B., Verguts, T., \& Van den Bussche, E. (2017). Beyond trial-bytrial adaptation: A quantification of the time scale of cognitive control. Journal of Experimental Psychology: Human Perception and Performance, 43, 509-517. doi: 10.1037/xhp0000324 Www

Allport, D. A., Styles, E. A., \& Hsieh, S. (1994). Shifting intentional set: Exploring the dynamic control of tasks. In C. Umilta \& M. Moscovitch (Eds.), Conscious and nonconscious information processing: Attention and performance XV (pp. 421-452). Cambridge, MA: MIT Press.

Arrington, C. M., \& Logan, G. D. (2004). The cost of a voluntary task switch. Psychological Science, 15, 610-615. doi: 10.1111/j.09567976.2004.00728.x WWW

Asch, S. E. (1956). Studies of independence and conformity: I. A minority of one against a unanimous majority. Psychological Monographs: General and Applied, 70, 1-70. doi: 10.1037/ h0093718

Badre, D. (2008). Cognitive control, hierarchy, and the rostrocaudal organization of the frontal lobes. Trends in Cognitive Sciences, 12, 193-200. doi: 10.1016/j.tics.2008.02.004 WWW

Badre, D., \&Wagner, A.D. (2006). Computational and neurobiological mechanisms underlying cognitive flexibility. Proceedings of the National Academy of Sciences of the United States of America, 103, 7186-7191. doi: 10.1073/pnas.0509550103 |WW

Braem, S., Abrahamse, E. L., Duthoo, W., \& Notebaert, W. (2014). What determines the specificity of conflict adaptation? A review, critical analysis, and proposed synthesis. Frontiers in Psychology, 5:1134. doi: 10.3389/fpsyg.2014.01134 WWW

Csíkszentmihályi, M. (1996). Creativity: Flow and the psychology of discovery and invention. New York, NY: Harper Collins.

Darley, J. M., \& Latane, B. (1968). Bystander intervention in emergencies: Diffusion of responsibility. Journal of Personality and Social Psychology, 8, 377-383. doi: 10.1037/h0025589 WWW

Dovidio, J. F., Piliavin, J. A., Schroeder, D. A., \& Penner, L. (2006). The social psychology of prosocial behavior. Mahwah, NJ: Lawrence Erlbaum Associates Publishers.

Dreisbach, G. (2012). Mechanisms of cognitive control: The functional role of task rules. Current Directions in Psychological Science, 21, 227-231. doi: 10.1177/0963721412449830

Dreisbach, G., \&Fischer, R. (2012). Conflicts as aversive signals. Brain and Cognition, 78, 94-98. doi: 10.1016/j.bandc.2011.12.003 WWW 
Dreisbach, G., Goschke, T., \& Haider, H. (2007). The role of task rules and stimulus-response mappings in the task switching paradigm. Psychological Research, 71, 383-392. doi: 10.1007/ s00426-005-0041-3 WWW

Duran, N. D., Dale, R., \& McNamara, D. S. (2010). The action dynamics of overcoming the truth. Psychonomic Bulletin \& Review, 17, 486-491. doi: 10.3758/PBR.17.4.486 WWW

Everett, J. A., Pizarro, D. A., \& Crockett, M. J. (2016). Inference of trustworthiness from intuitive moral judgments. Journal of Experimental Psychology: General, 145, 772. doi: 10.1037/

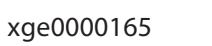

Foerster, A., Wirth, R., Kunde, W., \& Pfister, R. (2017). The dishonest mind set in sequence. Psychological Research, 81, 878-899. doi: 10.1007/s00426-016-0780-3 WwW

Fox, K. J. (1987). Real punks and pretenders: The social organization of a counterculture. Journal of Contemporary Ethnography, 16, 344-370. doi: 10.1177/0891241687163006

Gade, M., Schuch, S., Druey, M. D., \& Koch, I. (2014). Inhibitory control in task switching. In J. Grange \& G. Houghton (Eds.), Task switching and cognitive control (pp. 137-159). New York, NY: Oxford University Press.

Gade, M., Souza, A. S., Druey, M. D., \& Oberauer, K. (2017). Analogous selection processes in declarative and procedural working memory: $\mathrm{N}-2$ list-repetition and task-repetition costs. Memory \& Cognition, 45, 26-39. doi: 10.3758/s13421-0160645-4

Grange, J. A., \& Kowalczyk, A. (2017). The effect of aging on inhibition in task switching: Controlling for episodic retrieval. Manuscript submitted for publication.

Gratton, G., Coles, M. G., \& Donchin, E. (1992). Optimizing the use of information: Strategic control of activation of responses. Journal of Experimental Psychology: General, 121, 480-506. doi: 10.1037/0096-3445.121.4.480 |wWw

Hasson, U., Simmons, J. P., \& Todorov, A. (2005). Believe it or not: On the possibility of suspending belief. Psychological Science, 16, 566-571. doi: 10.1111/j.0956-7976.2005.01576.x WWW

Hoffman, M. L. (1981). Is altruism part of human nature? Journal of Personality and Social Psychology, 40, 121-137. doi: 10.1037/0022-3514.40.1.121

Hsieh, S., Chang, C. C., \& Meiran, N. (2012). Episodic retrieval and decaying inhibition in the competitor-rule suppression phenomenon. Acta Psychologica, 141, 316-321. doi: 10.1016/j. actpsy.2012.09.001 |wWw

Jusyte, A., Pfister, R., Mayer, S. V., Schwarz, K. A., Wirth, R., Kunde, W., \& Schöneberg, M. (2017). Smooth criminal: Convicted rule-breakers show reduced cognitive conflict during deliberate rule violations. Psychological Research, 81, 939-946. doi: 10.1007/s00426-016-0798-6 WW

Kessler, Y., Shencar, Y., \& Meiran, N. (2009). Choosing to switch: Spontaneous task switching despite associated behavioral costs. Acta Psychologica, 131, 120-128. doi: 10.1016/j. actpsy.2009.03.005 WWW
Koch, I., Gade, M., Schuch, S., \& Philipp, A. M. (2010). The role of inhibition in task switching: A review. Psychonomic Bulletin \& Review, 17, 1-14. doi: 10.3758/PBR.17.1.1 WWW

Kool, W., McGuire, J. T., Rosen, Z. B., \& Botvinick, M. M. (2010). Decision making and the avoidance of cognitive demand. Journal of Experimental Psychology: General, 139, 665. doi: 10.1037/a0020198 WWW

Kuhns, D., Lien, M. C., \& Ruthruff, E. (2007). Proactive versus reactive task-set inhibition: Evidence from flanker compatibility effects. Psychonomic Bulletin \& Review, 14, 977-983. doi: 10.3758/ BF03194131 |WW|

Logan, G. D., \& Zbrodoff, N. J. (1979). When it helps to be misled: Facilitative effects of increasing the frequency of conflicting stimuli in a Stroop-like task. Memory \& Cognition, 7, 166-174. doi: 10.3758/BF03197535

MATLAB and Statistics Toolbox Release 2014a, The MathWorks, Inc., Natick, Massachusetts, United States.

Mayr, U., \& Keele, S. W. (2000). Changing internal constraints on action: The role of backward inhibition. Journal of Experimental Psychology: General, 129, 4-26. doi: 10.1037/0096-3445.129.1.4 WWW

Meiran, N., Hsieh, S., \& Dimov, E. (2010). Resolving task rule incongruence during task switching by competitor rule suppression. Journal of Experimental Psychology: Learning, Memory, and Cognition, 36, 992-1002. doi: 10.1037/a0019761 |WW

Milgram, S. (1963). Behavioral study of obedience. Journal of Abnormal and Social Psychology, 67, 371-378. doi: 10.1037/ h0040525 WWW

Monsell, S. (2003). Task switching. Trends in Cognitive Sciences, 7, 134-140. doi: 10.1016/S1364-6613(03)00028-7 |wWW

Notebaert, W., \& Verguts, T. (2008). Cognitive control acts locally. Cognition, 106,1071-1080.doi:10.1016/j.cognition.2007.04.011 WWW

Paunonen, S. V. (2006). You are honest, therefore I like you and find you attractive. Journal of Research in Personality, 40, 237249. doi: 10.1016/j.jrp.2004.12.003

Pfister, R., \& Janczyk, M. (2013). Confidence intervals for two sample means: Calculation, interpretation, and a few simple rules. Advances in Cognitive Psychology, 9, 74-80. doi: 10.2478/ v10053-008-0133-x WWW

Pfister, R., Wirth, R., Schwarz, K. A., Foerster, A., Steinhauser, M., $\&$ Kunde, W. (2016). The electrophysiological signature of deliberate rule violations. Psychophysiology, 53, 1870-1877. doi: 10.1111/psyp.12771

Pfister, R., Wirth, R., Schwarz, K. A., Steinhauser, M., \& Kunde, W. (2016). Burdens of non-conformity: Motor execution reveals cognitive conflict during deliberate rule violations. Cognition, 147, 93-99. doi: 10.1016/j.cognition.2015.11.009 WwW

Rogers, R. D., \& Monsell, S. (1995). Costs of a predictable switch between simple cognitive tasks. Journal of Experimental Psychology: General, 124, 207-231. doi: 10.1037/0096-3445 .124.2.207 
Scherbaum, S., Dshemuchadse, M., Fischer, R., \& Goschke, T. (2010). How decisions evolve: The temporal dynamics of action selection. Cognition, 115, 407-416. doi: 10.1016/j. cognition.2010.02.004 WWW

Schuch, S., \& Koch, I. (2004). The costs of changing the representation of action: Response repetition and responseresponse compatibility in dual tasks. Journal of Experimental Psychology: Human Perception and Performance, 30, 566-582. doi: 10.1037/0096-1523.30.3.566 [WwW

Serota, K. B., \& Levine, T. R. (2014). A few prolific liars: Variation in the prevalence of lying. Journal of Language and Social Psychology, 34, 138-157. doi: 10.1177/0261927X14528804

Simon, J. R. (1990). The effects of an irrelevant directional cue on human information processing. Advances in Psychology, 65, 31-86. doi: 10.1016/S0166-4115(08)61218-2

Spence, S. A., Farrow, T. F. D., Herford, A. E., Wilkinson, I. D., Zheng, Y., \& Woodruff, P. W. R. (2001). Behavioural and functional anatomical correlates of deception in humans. NeuroReport, 12, 2849-2853. doi: 10.1097/00001756-200109170-00019 [wWw

Torres-Quesada, M., Funes, M. J., \& Lupiáñez, J. (2013). Dissociating proportion congruent and conflict adaptation effects in a Simon-Stroop procedure. Acta Psychologica, 142, 203-210. doi: 10.1016/j.actpsy.2012.11.015 www
Van Steenbergen, H., Band, G. P., \& Hommel, B. (2009). Reward counteracts conflict adaptation evidence for a role of affect in executive control. Psychological Science, 20, 1473-1477. doi: 10.1111/j.1467-9280.2009.02470.x $\overline{w W w \mid}$

Van Steenbergen, H., Band, G. P., \& Hommel, B. (2010). In the mood for adaptation: How affect regulates conflictdriven control. Psychological Science, 21, 1629-1634. doi: 10.1177/0956797610385951 www

Wirth, R., Foerster, A., Rendel, H., Kunde, W., \& Pfister, R. (2017). Rule-violations sensitize towards authority-related stimuli. Cognition \& Emotion, 1-14. Advance online publication. doi: 10.1080/02699931.2017.1316706 WWW

Wirth, R., Pfister, R., Foerster, A., Huestegge, L., \& Kunde, W. (2016). Pushing the rules: Effects and aftereffects of deliberate rule violations. Psychological Research, 80, 838-852. doi: 10.1007/ s00426-015-0690-9 Www

Wirth, R., Pfister, R., \&, Kunde W. (2016). Asymmetric transfer effects between cognitive and affective task disturbances. Cognition \& Emotion, 30, 399-416. doi: 10.1080/02699931.2015.1009002 WWW

Yinger, J. M. (1982). Countercultures: The promise and peril of a world turned upside down. New York, NY: Simon and Schuster.

RECEIVED 29.05.2017| ACCEPTED 21.06.2018 